\title{
Mitochondrial Haplotype Analysis as a Tool for Differentiating Isolates of Verticillium dahliae
}

\author{
Frank N. Martin
}

First author: U.S. Department of Agriculture-Agricultural Research Service, 1636 East Alisal St., Salinas, CA 93905. Accepted for publication 15 July 2010.

\begin{abstract}
Martin, F. N. 2010. Mitochondrial haplotype analysis as a tool for differentiating isolates of Verticillium dahliae. Phytopathology 100:12311239.

The ability to monitor mitochondrial background in Verticillium dahliae may provide an additional tool for population studies and monitoring clonal populations. Published mitochondrial genome sequences of $V$. dahliae (DQ351941) were used to design primers for amplification of spacer regions for assessment of mitochondrial haplotype differences among isolates. Five regions were examined $(5,229 \mathrm{bp}$, or $19 \%$ of the total genome size) for 30 isolates representing a range in vegetative compatibility group (VCG), host, and geographic origin. Observed differences among isolates were due to single nucleotide polymorphisms, different numbers of bases in specific homopolymeric regions, and copies of subrepeated sequences. When the differences observed for each locus were totaled there were 28 total groupings; when the results of each locus for individual isolates were combined there were 15 mitochondrial haplotypes. Some of the observed groupings correlated with VCG. For example, five VCG-1A and VCG-1B isolates from California, Spain, and

Greece had identical haplotypes; however, this was not observed for VCG-2 or VCG-4 isolates. While some VCG-2 isolates also were identical and fell into a single haplotype, five haplotypes were found for this group (five other haplotypes were observed for other isolates that had not been characterized to VCG but grouped with VCG-2 isolates in the phylogenetic analysis). Likewise, five VCG-4 isolates fell into four mitochondrial haplotypes, one of which was identical to the largest VCG2 grouping. A heterokaryon self-incompatible isolate that was reported in the literature to cluster with VCG-2 isolates by amplified fragment length polymorphism analysis was identical with VCG-1 isolates for four of the five loci, but was intermediate between VCG-1 and VCG-2 in the haplotype analysis. Phylogenetic analysis with these regions revealed the mitochondrial background of VCG-1 and VCG2-B to be monophyletic but VCG-2A and VCG-4 could not be separated. The results obtained indicate that there is variation in mitochondrial haplotypes and this type of analysis may be a useful for characterization of isolates. While data from all five regions was used for the haplotype separation in this study, depending on the VCG or the level of variability observed within a population it is possible to use fewer loci.
\end{abstract}

Given its broad host range and wide distribution Verticillium dahliae Kleb. is an important vascular wilt pathogen of a number of economically important crop plants. It can survive for long periods in the soil as microsclerotia, which can germinate and systemically infect host roots when conditions are favorable. While some level of association of isolates with a specific host has been observed, for the most part there is limited host specificity among strains $(4,14,15,37)$. A sexual stage has not been observed and amplified fragment length polymorphism (AFLP) analysis of a number of isolates suggests clonal reproduction in field populations (10), although more recent microsatellite data suggest that some level of recombination and gene flow has occurred in field populations at some point in time (1).

Given the limited variation in morphological features and broad host range with limited host specificity one of the primary means for characterization of isolates has been classification into vegetative compatibility groups (VCGs) using nitrate nonutilizing mutants $(2,6,16,18,19,40)$. Presently there are six VCGs with the most common ones VCG-1, VCG-2, and VCG-4. Based on the level of complementation with standard tester isolates these VCGs are further subdivided into A and B subgroups (2,17,39), with VCG-2B isolates from artichoke in Spain further subdivided into

Corresponding author: F. N. Martin; E-mail address: frank.martin@ars.usda.gov

* The $\boldsymbol{e}$-Xtra logo stands for "electronic extra" and indicates that the online version contains two supplemental files.

doi:10.1094/PHYTO-12-09-0352

This article is in the public domain and not copyrightable. It may be freely reprinted with customary crediting of the source. The American Phytopathological Society, 2010.
VCG2Ba and VCG-2Br based on the presence/absence of specific polymerase chain reaction (PCR) amplified markers (10). Isolates of VCG-3, VCG-5, and VCG-6 are less frequently encountered $(3,19,40)$. While VCG analysis can provide useful information about an isolate, it is a time consuming process to generate and test isolates. Furthermore, some isolates are heterokaryon selfincompatible and thus, cannot be classified into VCG. While isolates in different VCGs are believed to be genetically isolated, each VCG contains genotypically different isolates and do not represent a clonal population $(1,9,10)$.

In an effort to simplify isolate identification and differentiation into populations a variety of molecular methods have been used to characterize isolates. This includes restriction fragment length polymorphism (RFLP) analysis using clones of genomic DNA as probes $(5,13,30,31)$, PCR-RFLP of regions of the rDNA $(32,41)$ or the mitochondrially encoded small ribosomal subunit of the rDNA (32), RAPD analysis $(3,4,7,13,20-22,44)$, as well as sequence differences in the internal transcribed spacer (ITS) region (28) and intergenic region $(29,36,37)$ of the rDNA. Several primer pairs for amplification of specific sized fragments also have been used to characterize isolates into specific subgroups, some of which are associated with defoliating characteristics of the isolates $(10,11,25)$. Consistent correlations between groupings obtained from these molecular analyses and other characteristics such as VCG, host of recovery, or geographic region were not observed. However, Collado-Romero et al. (10) used AFLP analysis to examine a large collection of isolates predominantly from the Mediterranean region and found that isolates grouped according to VCG, regardless of which host the isolates were recovered from or their geographic origin. 
Limited information on the phylogenetic relationships among VCGs of $V$. dahliae is available. The grouping of VCGs in the AFLP analysis of Collado-Romero et al. (10) suggested an affinity between VCG-1A and B isolates and one subgroup of VCG-2B isolates while VCG-4B isolates clustered with VCG-2A and another subgroup of VCG-2B isolates. This relationship was explored further by combining the results of the AFLP analysis with sequence data from six nuclear loci to evaluate phylogenetic relationships (9). Similar results were obtained; there were two main lineages with one comprised of VCG-1A and B isolates and one subgroup of VCG-2B isolates while the other had two primary clades, one with VCG-2A and VCG-4B isolates and the other with VCG-4A, VCG-6, and the other subgroup of VCG-2B isolates. One of the limiting factors for conducting this type of phylogenetic analysis is the availability of suitable loci that provide the appropriate discrimination among VCGs.

In clonally reproducing populations of the pathogen differences in mitochondrial DNA should be conserved within a lineage and may be useful as a marker to help characterize populations as has been reported for Phytophthora ramorum (24). A cytoplasmically encoded marker would also compliment the information obtained with nuclear loci and be useful for investigations to evaluate if there were recombining populations. Sequence differences in the mitochondrially encoded small ribosomal subunit that separated a sunflower isolate from other isolates have been identified using PCR-RFLP analysis (32). The mitochondrial genome for one isolate of $V$. dahliae has been sequenced and several intergenic regions were used for developing markers to differentiate species of Verticillium (33). This mitochondrial genomic sequence can be used to design primers to look for polymorphisms in intervening regions between genes that would likely exhibit greater intraspecific variation among isolates than coding regions. The objective of this project was to explore this possibility with a geographically diverse range of isolates that have been characterized in other studies by VCG, RAPD, and AFLP analysis, or sequence analysis of nuclear encoded loci $(4,9,10,37)$.

\section{MATERIALS AND METHODS}

Fungal material. Isolates used in this investigation are listed in Table 1 with DNA for the indicated isolates from prior publications $(9,10)$ provided by R. M. Jiménez-Díaz. Cultures of $V$. dahliae for DNA extraction were grown in potato dextrose broth (Difco, Detroit, MI) for 4 to 6 days at room temperature prior to DNA extraction using a FastDNA kit (Qbiogene, Irvine, CA). DNA quality was assessed by gel electrophoresis and quantity with a Nanodrop spectrophotometer (Nanodrop Technologies, Wilmington, DE).

Design of primers. The mitochondrial genome of $V$. dahliae (DQ 351941) reported by Pantou et al. (33) was used for designing primers for the regions evaluated in this investigation (Table 2). Intervening regions between nad3 to nadl (543 bp, region 1), atp6 to ribosomal small subunit (384 bp, region 2), cox3 to nad6 (1,632 bp, region 3), cob to coxl (1,157 bp, region $4)$, and coxl to larger ribosomal subunit $(1,483 \mathrm{bp}$, region 5) were examined. While predominantly spacer sequences, tRNAs were

TABLE 1. Isolates of Verticillium dahliae used in this investigation and their mitochondrial haplotypes

\begin{tabular}{|c|c|c|c|c|c|c|c|c|c|c|}
\hline \multirow{2}{*}{$\begin{array}{l}\text { Isolate } \\
\text { number }\end{array}$} & \multirow[b]{2}{*}{$\mathrm{VCG}^{\mathrm{a}}$} & \multirow[b]{2}{*}{ Host } & \multirow{2}{*}{$\begin{array}{l}\text { Isolation } \\
\text { location }\end{array}$} & \multicolumn{5}{|c|}{ Mitochondrial region $^{\mathrm{b}}$} & \multirow[b]{2}{*}{ Haplotype $^{\mathrm{c}}$} & \multirow{2}{*}{$\begin{array}{l}\text { Molecular grouping } \\
\text { and reference }\end{array}$} \\
\hline & & & & 1 & 2 & 3 & 4 & 5 & & \\
\hline Gh101e & VCG-1 & G. hirsutum (cotton) & California & A & A & $\mathrm{A}$ & A & A & I & $V d \mathrm{NC}$ II $(3,37)$ \\
\hline V137 I ${ }^{\mathrm{f}}$ & VCG-1A & O. europaea (olive) & Spain & A & $\mathrm{A}$ & $\mathrm{A}$ & A & A & I & $1 \alpha(10)$ \\
\hline V403 $\mathrm{I}^{\mathrm{f}}$ & VCG-1A & C. scolynus (artichoke) & Spain & A & A & A & A & A & I & $1 \alpha(9,10)$ \\
\hline V854 $\mathrm{I}^{\mathrm{f}}$ & VCG-1A & G. hirsutum & Spain & A & A & A & A & A & I & $1 \alpha(10)$ \\
\hline V661 If & VCG-1B & G. hirsutum & Greece & A & $\mathrm{A}$ & $\mathrm{A}$ & A & A & I & $1 \alpha /-(9,10)$ \\
\hline V537 If & HSI & C. scolynus & Spain & A & A & B & A & A & II & $1 \alpha(9,10)$ \\
\hline Fca21 ${ }^{\mathrm{e}}$ & VCG-2 & Fragariae $\times$ ananassa & California & B & B & B & B & B & III & $V d \mathrm{NC} I(3,37)$ \\
\hline Fca29e & - & Fragariae $\times$ ananassa & California & B & B & B & B & B & III & $V d \mathrm{NC}$ I (37) \\
\hline Cv79e & - & C. vulgaris (watermelon) & California & $\mathrm{B}$ & B & $\mathrm{B}$ & B & $\mathrm{B}$ & III & $V d \mathrm{NC} I(37)$ \\
\hline $\mathrm{Cv} 85^{\mathrm{e}}$ & VCG-2 & C. vulgaris & California & B & B & B & B & B & III & $V d \mathrm{NC}$ I $(3,37)$ \\
\hline $\mathrm{Cs} 80^{\mathrm{e}}$ & VCG-2 & C. scolynus & California & $\mathrm{B}$ & $\mathrm{B}$ & $\mathrm{B}$ & $\mathrm{B}$ & B & III & $V d \mathrm{NC} I(3,37)$ \\
\hline $\mathrm{Ls} 331^{\mathrm{e}}$ & - & L. sativa (lettuce) & California & $\mathrm{B}$ & $\mathrm{B}$ & $\mathrm{A}$ & $\mathrm{B}$ & $\mathrm{B}$ & IV & $V d \mathrm{NC} I(37)$ \\
\hline V548 If & VCG-2A & C. scolynus & Spain & B & B & B & B & B & III & $2 \alpha \mathrm{III}(10)$ \\
\hline V717 If & VCG-2A & O. europaea & Italy & B & B & B & B & B & III & $2 \alpha \operatorname{III}(10)$ \\
\hline V364 If & VCG-2A & O. europaea & Syria & B & B & B & B & B & III & R. M. Jiménez-Díaz \\
\hline V800 If & VCG-2A & O. europaea & Spain & $\mathrm{B}$ & $\mathrm{B}$ & B & $\mathrm{B}$ & $\mathrm{B}$ & III & $2 \alpha \operatorname{III}(9,10)$ \\
\hline Mp 89e & VCG-2 & Mentha $\times$ piperita $($ mint $)$ & Oregon & $\mathrm{C}$ & $\mathrm{C}$ & $\mathrm{C}$ & $\mathrm{C}$ & $\mathrm{C}$ & $\mathrm{V}$ & $V d \mathrm{NC}$ III $(3,37)$ \\
\hline V396 If & VCG-2B & C. scolynus & Spain & $\mathrm{B}$ & $\mathrm{C}$ & $\mathrm{D}$ & $\mathrm{D}$ & $\mathrm{C}$ & VI & $2 \beta^{824}$ II $(9,10)$ \\
\hline V539 $\mathrm{I}^{\mathrm{f}}$ & VCG-2B & C. scolynus & Spain & $\mathrm{B}$ & $\mathrm{C}$ & $\mathrm{E}$ & $\mathrm{C}$ & $\mathrm{D}$ & VII & $2 \beta^{334}(10)$ \\
\hline $\mathrm{Cf} 40^{\mathrm{e}}$ & - & C. апnиum (chile pepper) & California & B & $\mathrm{C}$ & $\mathrm{C}$ & $\mathrm{E}$ & $\mathrm{E}$ & VIII & $V d \mathrm{NC}$ II (37) \\
\hline $\mathrm{Cf}-45^{\mathrm{e}}$ & - & C. аппиит (chili pepper) & California & $\mathrm{B}$ & $\mathrm{C}$ & $\mathrm{C}$ & $\mathrm{C}$ & $\mathrm{C}$ & IX & $V d \mathrm{NC}$ II (37) \\
\hline $\mathrm{Ls} 7^{\mathrm{e}}$ & VCG-2 & L. sativa & California & B & $\mathrm{C}$ & $\mathrm{C}$ & B & B & $\mathrm{X}$ & $V d \mathrm{NC} I(3,37)$ \\
\hline $\operatorname{Ls} 17^{\mathrm{e}}$ & - & L. sativa & California & $\mathrm{B}$ & $\mathrm{B}$ & B & $\mathrm{D}$ & $\mathrm{C}$ & XI & $V d \mathrm{NC}$ III (37) \\
\hline $\mathrm{Ca} 63^{\mathrm{e}}$ & - & C. апnиит (bell pepper) & California & $\mathrm{B}$ & $\mathrm{C}$ & $\mathrm{C}$ & $\mathrm{C}$ & $\mathrm{C}$ & IX & $V d \mathrm{NC}$ II (37) \\
\hline $\operatorname{Sm} 113^{\mathrm{e}}$ & VCG-4 & S. melongena (eggplant) & California & $\mathrm{B}$ & $\mathrm{B}$ & $\mathrm{B}$ & $\mathrm{B}$ & $\mathrm{F}$ & XII & $V d \mathrm{NC}$ III $(3,37)$ \\
\hline Le88 & VCG-4 & L. esculentum & California & B & B & B & B & B & III & $V d \mathrm{NC} I(3,37)$ \\
\hline St91 ${ }^{\mathrm{e}}$ & VCG-4 & S. tuberosum (potato) & Oregon & $\mathrm{D}$ & B & B & B & $\mathrm{F}$ & XIII & $V d \mathrm{NC}$ III $(3,37)$ \\
\hline V310 If & VCG-4B & G. hirsutum & Israel & $\mathrm{B}$ & $\mathrm{B}$ & $\mathrm{B}$ & $\mathrm{B}$ & $\mathrm{G}$ & XIV & $4 \beta$ II (10) \\
\hline V792 I & VCG-4B & O. europaea & Spain & B & $\mathrm{B}$ & $\mathrm{B}$ & $\mathrm{B}$ & $\mathrm{G}$ & XIV & $4 \beta$ II (10) \\
\hline DQ351941g & - & - & - & $\mathrm{E}$ & $\mathrm{C}$ & $\mathrm{F}$ & $\mathrm{F}$ & $\mathrm{H}$ & $\mathrm{XV}$ & Pantou et al. (33) \\
\hline
\end{tabular}

a VCG = vegetative compatibility group as reported in cited references. HSI = heterokaryon self-incompatible, “_” indicates unknown.

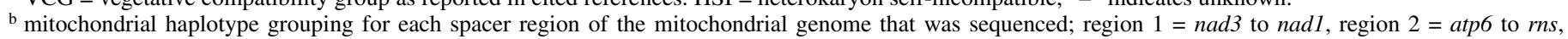
region $3=\operatorname{cox} 3$ to nad6, region $4=\operatorname{cob}$ to $\operatorname{cox} 1$, and region $5=\operatorname{cox} 1$ to $\mathrm{rnl}$.

c Final mitochondrial haplotype based on the summation of results for regions 1 through 5 .

${ }^{\mathrm{d}}$ Molecular grouping of isolate in cited reference where prior results on VCG or nuclear genotype reported.

e Cultures obtained from K. V. Subbarao.

${ }^{\mathrm{f}}$ Extracted DNA samples were provided by R. M. Jiménez-Díaz.

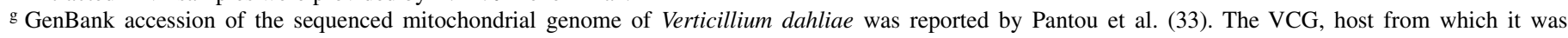
recovered, and location of recovery was not indicated. 
encoded in some of these regions (3, 4, 1, and 1 in regions 1, 3, 4, and 5, respectively). Primers were manually designed to amplify at the same annealing temperature and magnesium concentration to simplify template amplification. The intergenic sequence between $\operatorname{nad} 3$ and $n a d 1$ (region 1) is one of the regions Pantou et al. (33) used to differentiate Verticillium spp.

Template amplification and sequencing. Primer pairs used for template amplification are listed in Table 2. All amplifications were done using approximately $10 \mathrm{ng}$ of template DNA, $0.5 \mathrm{mM}$ forward and reverse primers, $2 \mathrm{mM} \mathrm{MgCl}_{2}, 100 \mu \mathrm{M} \mathrm{dNTP}, 1 \times$ amplification buffer, and 1 unit of AmpliTaq (Applied Biosystems, Foster City, CA) in a volume of $25 \mu \mathrm{l}$. Templates were amplified in an ABI 9600 thermal cycler with the following cycling conditions: 1 interval of $95^{\circ} \mathrm{C}$ for $3 \mathrm{~min} ; 35$ cycles of $95^{\circ} \mathrm{C}$ for $1 \mathrm{~min}, 57^{\circ} \mathrm{C}$ for $2 \mathrm{~min}$ and $72^{\circ} \mathrm{C}$ for $1 \mathrm{~min} ; 1$ interval of $72^{\circ} \mathrm{C}$ for $5 \mathrm{~min}$ followed by a $4^{\circ} \mathrm{C}$ hold. After confirming template amplification by running samples on an agarose gel, sequencing templates were prepared by treatment with ExoSap (USB, Cleveland, $\mathrm{OH}$ ) in accordance with the manufacturer's instructions and sent to the Nucleic Acid Sequencing Facility at the Penn State University (University Park) for sequencing with the amplification primers unless otherwise noted (Table 2). Each template was sequenced in both directions to generate a consensus sequence based on complementary strands. Sequencher 4.7 (Gene Codes, Ann Arbor, MI) was used to edit the sequences and generate consensus sequences while DS Gene v2.5 (Accelrys, San Diego, CA) was used for editing and making alignments. Sequence trace files were carefully examined to evaluate if multiple bases were present at a single locus (which would represent the presence of more than one form of mitochondrial genome).

Haplotype analysis. Sequences for each region were aligned and flanking coding regions were removed due to a high level of sequence conservation. The only exception was for region 4, where 29 bp of the $3^{\prime}$ end of the $c o b$ gene was retained due to the presence of a single nucleotide polymorphism (SNP) in isolates Ls7 and V396 I. Polymorphisms among the isolates in the align- ments of each region individually were noted and classified with a letter designation; examples for each type have been deposited in GenBank (Table 3). Once the analysis of the individual loci was completed the results for all 5 loci were combined and a Roman numeral designation for each final haplotype was given (Table 1). DnaSP version 5.10.01 (23) was used to confirm the haplotype assignments. To visualize the relationships among the different haplotypes, individual sequences were concatenated in McClade version 4.02 (Sinaur Associates, Sunderland, MA) to create a single alignment file. To graphically show the differences among haplotypes unweighted pair-group method with arithmetic average (UPGMA) analysis was done using PAUP version 4.0b10 (Sinaur Associates) and to ensure length differences due to indels were included in the resultant tree, gaps were treated as a character state. To graphically illustrate a network for the differences among the haplotypes, a concatenated sequence file with representation of each haplotype was analyzed using TCS version 1.21 (8).

Phylogenetic analysis. A partition homogeneity test was run in PAUP version $4.0 \mathrm{~b} 10$ to evaluate the homogeneity of the concatenated data set. Phylogenetic relationships were inferred using three different methods with the corresponding mitochondrial sequences from V. albo-atrum (accession ABPE01004148 from the V. albo-atrum genomic sequencing project; 42) used as an outgroup. Maximum parsimony (MP) analysis was done using PAUP version $4.0 \mathrm{~b} 10$ with a heuristic tree search with MULPARS on, steepest decent option off, random addition of sequences (10 replicates) and TBR branch swapping. To determine support for the various clades of the trees, the analysis was bootstrapped with 1,000 replicates. Maximum likelihood (ML) and Bayesian analysis (BA) was done using TOPALi version 2.5 (27) with JModelTest version 0.1.1 (35) used to determine the appropriate nucleotide substitution model. Based on the values of the Akaike Information Criterion, Bayesian Information Criterion and Decision Theory Performance Based Selection for the concatenated data set, the HKY nucleotide substitution model was selected. ML analysis was run in TOPALi using PhyML version

TABLE 2. Primer sequences used for amplification and sequencing of the mitochondrial spacer regions used in this investigation for determining mitochondrial haplotypes in Verticillium dahliae

\begin{tabular}{|c|c|c|c|c|c|c|}
\hline Locus & Start $^{\mathrm{a}}$ & End $^{\mathrm{a}}$ & Amplicon size & Spacer region & Amplification primer & Nested sequencing primers \\
\hline I & 7,731 & 8,362 & 632 & nad3 - nad1 & $\begin{array}{l}\text { Vd1F - AGATAGTAGACAATCTCTTAC } \\
\text { Vd1R - TCTAATATAGATAAAAGAGTAGA }\end{array}$ & \\
\hline III & 15,283 & 17,045 & 1,763 & $\operatorname{cox} 3-\operatorname{nad} 6$ & $\begin{array}{l}\text { Vd3F - ACATTTTGTAGACGTTGTTTG } \\
\text { Vd3R - ATACAATATCTAACATACCTGT }\end{array}$ & $\begin{array}{l}\text { Vd3F2 - GAGTTGATGATCATCAACCT } \\
\text { Vd3R2 - TTTTAGTTTGAGGTTGATGAT } \\
\text { Vd3R3- CTAGGTATTTAGCTTCTTCTC } \\
\text { Vd3F3 - GACATGGAGAACAAGCTTC }\end{array}$ \\
\hline IV & 22,973 & 24,283 & 1,311 & $\operatorname{cob}-\operatorname{cox} 1$ & $\begin{array}{l}\text { Vd4F - CCTGTAGTTAGTGTAACAGA } \\
\text { Vd4R - ATAACCCTGAGAATAAAGCAA }\end{array}$ & $\begin{array}{l}\text { Vd4F2 - CATGAGTTGTGCATATAGATG } \\
\text { Vd4R2 - CAAAGTATAGAAAGCCATCTAT }\end{array}$ \\
\hline V & 25,747 & 164 & 1,602 & $\operatorname{cox} 1-\mathrm{rnl}$ & $\begin{array}{l}\text { Vd5F - AAACCTCACGCTTTTGTAAG } \\
\text { Vd5R - AAGTTACTAAGATATTTCAATTC }\end{array}$ & $\begin{array}{l}\text { Vd5F2 - CCAATATGCATAAATGGTTTAA } \\
\text { Vd5R2 - CTTGGAGCGATAAATGAGTA }\end{array}$ \\
\hline
\end{tabular}

${ }^{a}$ The start and end positions refer to the base positions in the sequenced mitochondrial genome DQ351941 of $V$. dahliae reported by Pantou et al. (33).

TABLE 3. GenBank accession numbers for spacer regions representing Verticillium dahliae mitochondrial haplotype classification

\begin{tabular}{|c|c|c|c|c|c|c|c|c|}
\hline Region & \multicolumn{8}{|c|}{ Haplotype } \\
\hline 1 (nad3 to $n a d 1)$ & GU291296 & GU291297 & GU291298 & GU291299 & DQ351941 & - & - & - \\
\hline $3(\cos 3$ to $n a d 6)$ & GU291303 & GU291304 & GU291305 & GU291306 & GU291307 & DQ351941 & - & - \\
\hline $4(\operatorname{cob}$ to $\operatorname{cox} 1)$ & GU291308 & GU291309 & GU291310 & GU291311 & GU291312 & DQ351941 & - & - \\
\hline $5(\operatorname{coxl}$ to $r n l)$ & GU291313 & GU291314 & GU291315 & GU291316 & GU291317 & GU291318 & GU291319 & DQ351941 \\
\hline
\end{tabular}


2.4.5 with 100 bootstrap replicates. BA was run in TOPALi using MrBayes version 3.1.1; two analyses were run simultaneously for 200,000 generations with a $10 \%$ sampling frequency and burn in of $25 \%$. The runs converged after 200,000 generations with the potential scale reduction factor having a value of 1.0. Data sets have been deposited in TreeBASE (S10602).

\section{RESULTS}

Polymorphisms in the amplified regions. Under the conditions specified, all five regions amplified well to produce amplicons of the size indicated in Table 2. Sequencing primers worked well for all templates with the exception of region 3; some of the internal primers had background problems with certain templates. The alignment for the spacer regions was $5,229 \mathrm{bp}$ in length with an average GC content of $26.8 \%$. Polymorphisms among isolates were due to SNPs, different numbers of bases in homopolymeric sequences (repeats of T), and different numbers of subrepeats. Among the 30 isolates included in the alignment there were 43 SNPs and 10 indels (which totaled $53 \mathrm{bp}$ ) for a total of 96 polymorphic sites. Excluding indels, Pi (per site nucleotide diversity $)=0.00256$. Alignments for all regions and a listing of specific sequence differences among haplotypes have been deposited in the supplemental files.

Region 1 (nad3 to nad1). A total of five subgroups were observed, with two positions where there was a homopolymeric string of 7, 8, or 9 "T"s depending on the isolate (subgroup A, B, and C; bases 25 to 26 and 437 to 438). One SNP also differentiated St91 (subgroup D; base 61) and another SNP differentiated DQ351941 (subgroup E; base 476) from other isolates.

Region 2 (atp6 to $r n s$ ). A total of 3 subgroups were observed, with a homopolymeric string of 8 or 10 "T"s at a single position (base 22-23) differentiating subgroup $\mathrm{A}$ and $\mathrm{C}$, respectively. A single SNP (base 255) further differentiated subgroup B from the other subgroup A isolates.

Region 3 (cox3 to nad6). This region had the most polymorphisms encountered and separated isolates into six subgroups. There were a total of 23 SNPs (nine unique to DQ351941) and a 15-bp insertion (subgroups A, C, D, E, and F; base 183 to 197), $12 \mathrm{bp}$ of which is a duplication of upstream sequences; these subgroups also shared an additional six SNPs. Subgroups C, D, E, and $\mathrm{F}$ also shared an additional five SNPs relative to the other subgroups with differences for subgroup $D$ and $E$ relative to subgroup C caused by a SNP at base 1113 and 1632, respectively.

Region 4 (cob to coxl). A total of six subgroups were observed based on seven SNPs. Group A (VCG-1) isolates all had 2 SNPs at base 870 to 871 of the intervening sequences (TT compared with AA for all other isolates). Subgroups C, D, E, and F also shared 2 SNPs (bases 193 and 378) with subgroups E and F separating from C due to a SNP at base 256 and 955 , respectively. In addition to polymorphisms in the sequences intervening between the cob to coxl genes, there was a synonymous substitution ( $\mathrm{G}$ for $\mathrm{T}$ ) $22 \mathrm{bp}$ from the termination codon of $\mathrm{cob}$ for isolates Ls17 and V396 I, which created a unique subgroup for these isolates (subgroup D) separating them from other subgroup $\mathrm{C}$ isolates.

Region 5 (coxl to $\mathrm{rnl}$ ). A total of eight subgroups were observed based on 12 SNPs (five unique to DQ531941), a homopolymeric string of 9 or 10 "T"s at a single position (base 1203) differentiating subgroups $C$ and $D$ from other subgroups, and a different copy number of a 15-bp imperfect repeated sequence. VCG-1 isolates (subgroup A) had only one copy of this subrepeat, subgroup $\mathrm{E}$ had three copies while subgroup B, C, D, $\mathrm{E}, \mathrm{G}$, and $\mathrm{H}$ had two copies with several SNPs in the imperfect repeat further differentiating them into three subgroups (B; G; and $\mathrm{C}, \mathrm{D}, \mathrm{E}$, and $\mathrm{H}$ ). Additional SNPs in other regions of the amplicon further differentiated these groupings.

Haplotype analysis. Combining the results of the separate loci for each isolate individually was done to determine the final haplotype classification of an isolate (Table 1). A total of 15 haplotypes were identified with some of the haplotype grouping corresponding to VCG. Haplotype I isolates were all VCG-1, however, greater variation was encountered in VCG-2 (five haplotypes with VCG-2A separate from VCG-2B) and VCG-4 isolates (four haplotypes). There were also five additional mitochondrial haplotypes for isolates that were not characterized for VCG but grouped with VCG-2 isolates in the UPGMA analysis (Fig. 1). Identity in haplotype between VCGs was also observed, as a VCG-4 isolate (Le88) and a number of VCG-2 isolates were Haplotype III. A heterokaryon self-incompatible isolate (V537I) was identical to Haplotype I isolates for four of the five loci and grouped intermediate between VCG-1 and VCG-2 isolates in the UPGMA analysis (Fig. 1). DQ531941 was more variable than the other isolates in four of the five loci and represented haplotype XV. A graphic representation of the differences among the haplotype networks was calculated by TCS version 1.2.1 (Fig. 2). Interestingly the lack of a 15-bp subrepeat repeat sequence in region 5 differentiated $\mathrm{H}$-I and $\mathrm{H}$-II from the rest of haplotypes while another deletion of $15 \mathrm{bp}$ in region 3 differentiated H-I from $\mathrm{H}-\mathrm{II}$ as well as the clade containing VCG-2B isolates (H-IV, $\mathrm{H}-\mathrm{V}, \mathrm{H}-\mathrm{VI}, \mathrm{H}-\mathrm{VII}, \mathrm{H}-\mathrm{VIII}, \mathrm{H}-\mathrm{IX}, \mathrm{H}-\mathrm{X}$, and $\mathrm{H}-\mathrm{XV}$ ) from the clade containing VCG-2A and VCG-4 isolates (H-III, H-XI, H-X11, $\mathrm{H}-\mathrm{XIII}$, and H-XIV). Both these length mutations are examples of homoplasy. There was no apparent correlation between geographic location of recovery and mitochondrial haplotype, as haplotype I and III had representatives from geographically diverse areas. Likewise isolates from the same VCG, host, and geographic location also exhibited variation in haplotype (artichoke isolates from Spain were haplotype VI and VII; the three lettuce isolates from California each were different haplotypes).

Phylogenetic analysis. The results of the maximum parsimony, maximum likelihood and Bayesian phylogenetic analysis of the combined dataset of region $1,2,4$, and 5 were similar with three primary clades observed: VCG-1 isolates and the heterokaryon self-incompatible isolate V537 I were on one clade; VCG-2B isolates on another clade and VCG-2A + VCG-4 isolates on the third (Fig. 3). The VCG-2A isolates did not exhibit variation while VCG-2B did. Likewise, two VCG-4B isolates (V301 I and V792 I) grouped separately from other VCG-4 isolates, which had limited (SM113, St91) or no (Le88) sequence variation with VCG-2A isolates. Isolates SM113 and St91were basal to the rest of the VCG-2A and VCG-4 clade, but strong bootstrap support for this position was not observed. The partition homogeneity test for the combined data set for regions $1,2,4$, and 5 was not significant $(P=0.16)$, but when region 3 was included in the combined data set the results were significant $(P=0.01)$, indicating incongruence in results among the different regions sequenced. The phylogenetic analysis using the concatenated data set including region 3 gave nearly identical results to those obtained in Figure 3 , the exception being isolate Ls7 grouped basal to V539 I and other VCG-2B isolates while isolate V537 I grouped with the large clade of VCG-2A and VCG-4 isolates.

\section{DISCUSSION}

The analysis of intergenic regions for five mitochondrial loci representing $19 \%$ of the total mitochondrial genome size differentiated 30 isolates of $V$. dahliae into 15 mitochondrial haplotypes. While five VCG-1 isolates from G. hirsutum, O. europaea, and C. scolynus from California, Spain, and Greece were monomorphic and different from all other isolates, a consistent association between mitochondrial haplotype and VCG was not observed for VCG-2 and VCG-4 (this comparison is incomplete as some of the cultures used in this investigation were not classified to VCG or VCG subgroups). Likewise, there was no consistent association among mitochondrial haplotype, host of 
isolation or geographic region of recovery of the isolates. Reports in the Verticillium literature on the correlation between molecular grouping and other characteristics of isolates have been inconsistent, with an association observed in some instances with geographic origin (31), host of recovery $(31,32,43)$, pathotype $(11,20,34,44)$, or VCG $(10,12,31,44)$, while other studies did not observe a correlation $(3-5,7,13,21,26,28-30,38,44)$.

In their comparison of AFLP grouping and VCG using a large collection of cultures, Collado-Romero et al. (10) reported that isolates grouped according to VCG independent of host and location of recovery. Twelve of the isolates from their study were also evaluated for mitochondrial haplotype in this investigation, and for 10 of them there was agreement in their groupings (Table 1, Fig. 1). The exceptions were isolates V539 I and V537 I, which in the AFLP analysis grouped closely together on the same clade, were positive for the 334-bp markers and were affiliated with VCG-1 isolates (with $100 \%$ bootstrap support for both groupings). However, these isolates had different mitochondrial haplotypes and while isolate V537 I grouped close to VCG-1 isolates, isolate V539 I was more distantly related clustering in with VCG2B isolates.

Qin et al. (37) sequenced the intergenic spacer (IGS) region of the rDNA for a range of isolates of $V$. dahliae and reported grouping of isolates into four main clades that had some

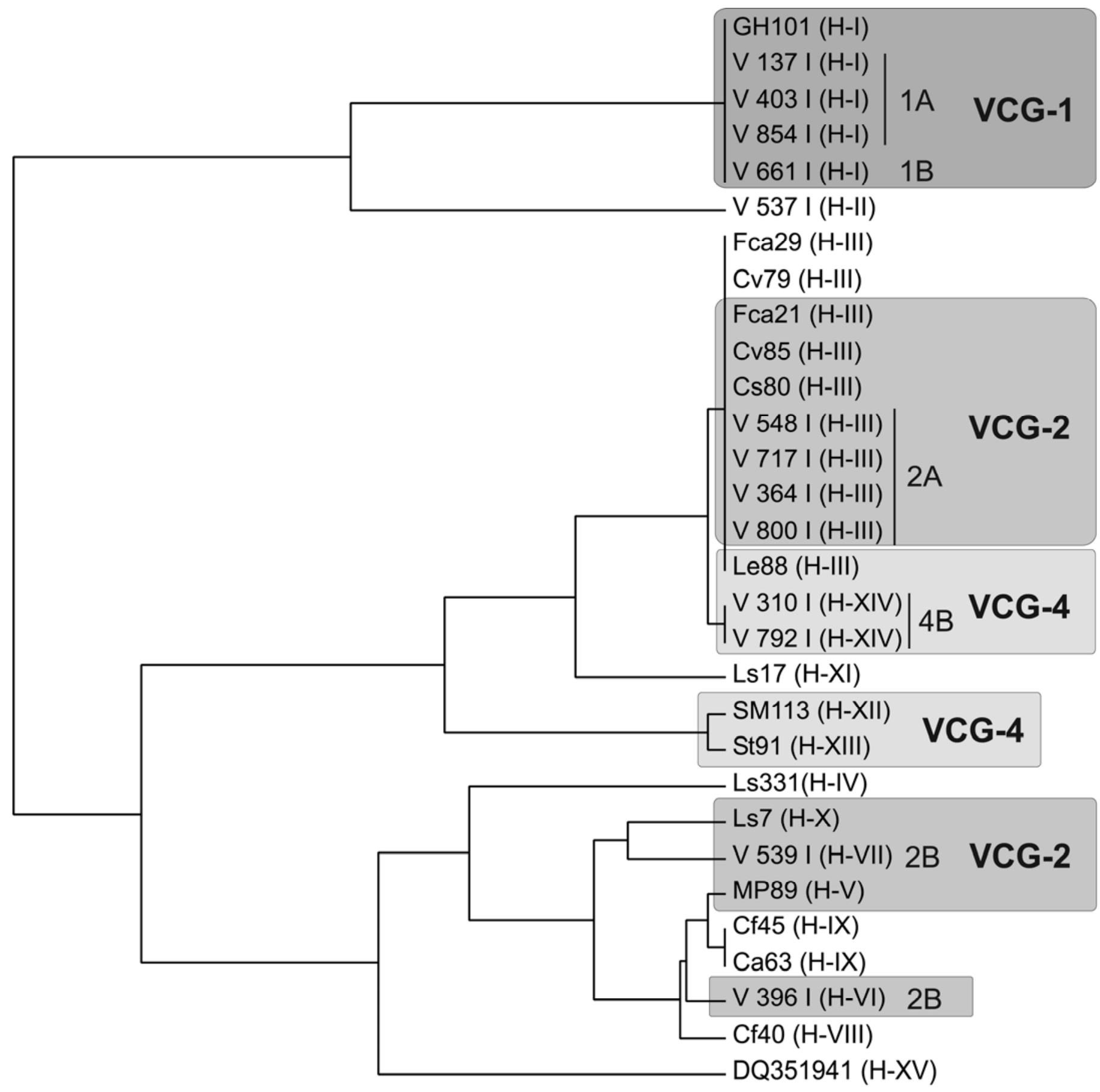

1 change

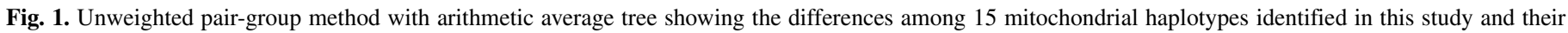

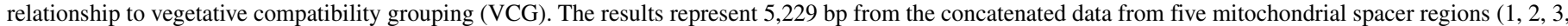

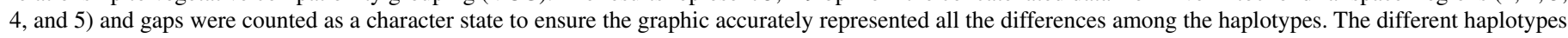
are represented by " $\mathrm{H}$ - Roman Numeral" following the isolate numbers. 
correlation to their host of origin (VCG was not included in the analysis). Sixteen of the isolates used in their study were also included in this investigation and while some similarities in mitochondrial haplotype were observed among isolates on specific clades from the IGS analysis, there were notable differences (Table 1). For example, eight isolates were examined from the IGS clade $V d \mathrm{NC}-1$ (primarily lettuce, strawberry and watermelon isolates) with six isolates exhibiting limited sequence differences in the IGS region yet these represented three mitochondrial haplotypes (H-III, H-IV, and H-X). Two isolates from clade $V d \mathrm{NC}-\mathrm{I}$ grouped together (Fca21 and Le88) and were more distantly related to the other isolates of this clade in the IGS analysis, but they did not differ in mitochondrial haplotype (H-III). Likewise, Cf40 and Mp89 exhibited limited IGS sequence divergence and were in clade $V d \mathrm{NC}$-III, yet had very different mitochondrial haplotypes (H-VIII and H-V, respectively). Differences in mitochondrial haplotypes for the remaining isolates corresponded to groupings in the IGS analysis (Ca63 and Cf45 were haplotype IX; SM113 and St91 were haplotype XII). Interestingly, Qin et al. (37) suggested that based on IGS sequence isolate Cf40 was the likely progenitor of isolate Ls17 recovered from lettuce, yet these isolates were very different in their mitochondrial haplotype

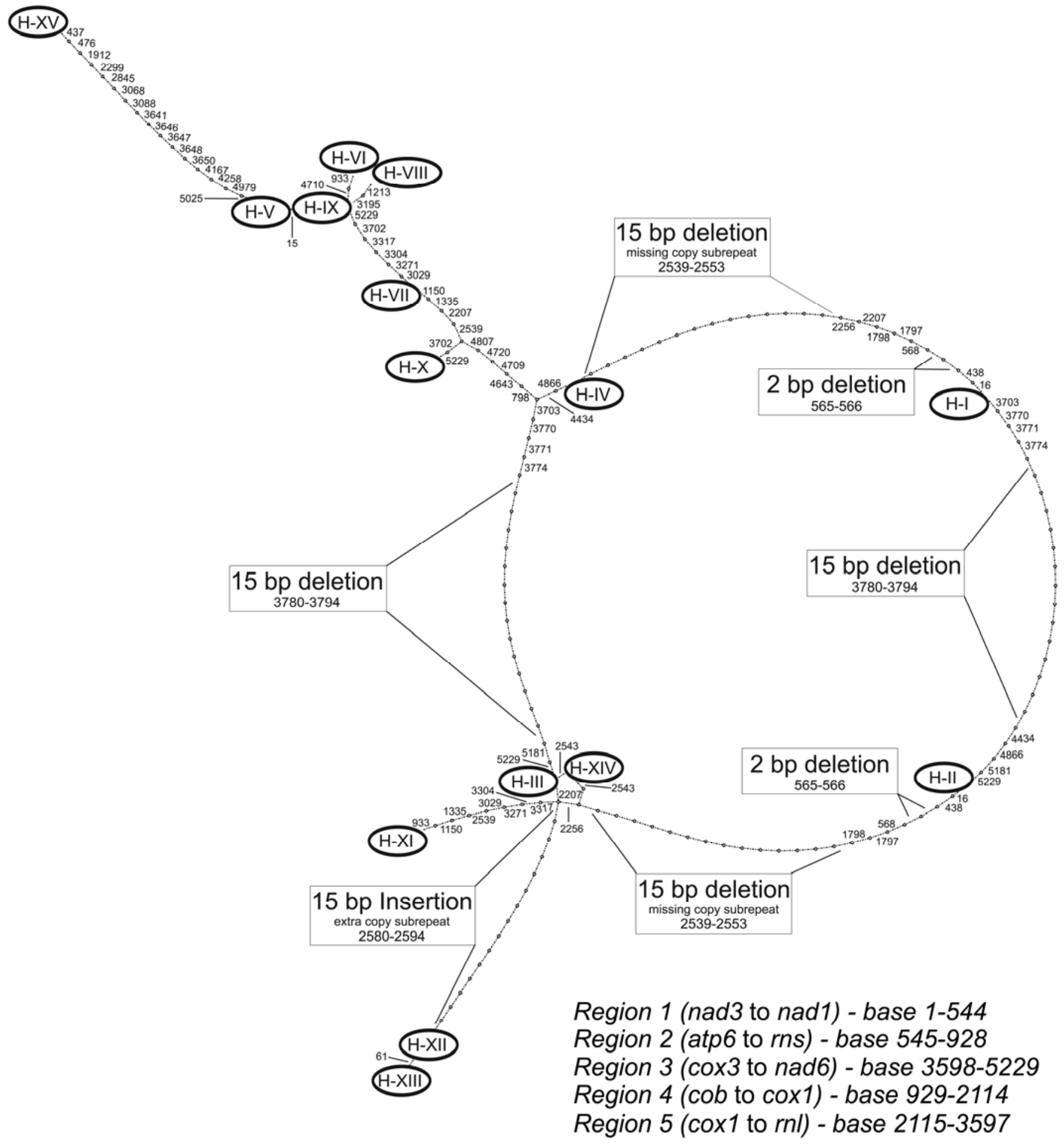

Fig. 2. Graphical representation of the relationships among mitochondrial haplotypes of Verticillium dahliae calculated by TCS version 1.21 (8). The different haplotypes are represented by " $\mathrm{H}$ - Roman Numeral" with base differences between them indicated by the base pair number from the concatenated alignment of the five sequenced regions indicated in the figure. 


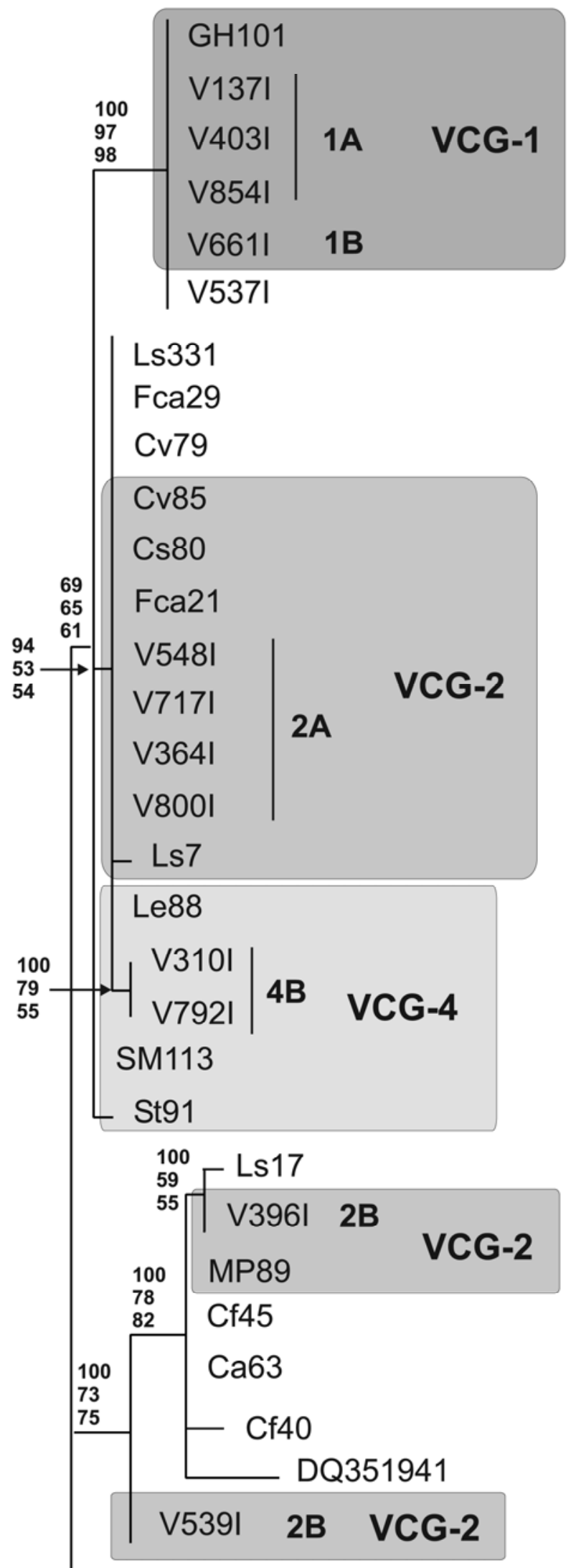

- 1 change

Fig. 3. Maximum parsimony tree using 3,598 bp from the concatenated data from four mitochondrial spacer regions $(1,2,4$, and 5$)$ inferred by a heuristic tree search with Verticillium albo-atrum selected as the outgroup. The numbers at the nodes are the percentage of trees that were supported by bootstrap analysis for maximum parsimony (1,000 replication, bottom number), maximum likelihood (100 replications, middle number), and percent Bayesian posterior probabilities (top number). DQ351941 is the GenBank accession for the mitochondrial genome sequence reported by Pantou et al. (33). The branch length for V. albo-atrum does not accurately reflect the level of sequence divergence in comparisons with $V$. dahliae. 
(dissimilar in four of five loci) suggesting they might not have as close an evolutionary relationship as suggested (assuming these represent clonally reproducing populations).

Using the mitochondrial spacer sequences for phylogenetic analysis generated trees with three groupings: VCG-1, VCG-2B, and VCG-2A and VCG-4 isolates. While this was similar to what has been reported for nuclear loci, there were also some differences. Collado-Romero et al. (9) examined the phylogenetic relationships among VCGs using AFLP data along with sequence data for 6 nuclear loci. Two major lineages were found, lineage I had VCG-1A, VCG-1B, and VCG-2B isolates positive for a 334-bp marker (with VCG-1 and -2 grouping separately) and lineage II had VCG-2A, VCG-2B isolates positive for an 824-bp marker, VCG-4A and VCG-4B isolates. Lineage II was further subdivided with VCG-2A and VCG-4B isolates in one clade and VCG-2B isolates positive for an 824-bp marker and VCG-4A in another. In the phylogenetic analysis of the mitochondrial sequences these two lineages were not clearly separated; VCG-1A and VCG-1B were identical but were separate from the VCG-2B isolate positive for the 334-bp marker (V539 I), and this isolate also clustered with good bootstrap support with the VCG-2B isolates positive for the 824-bp marker (V396 I). The VCG-2A isolates were not differentiated from three VCG-4 isolates although the VCG-4B isolates were. Doing the analysis with a combined dataset using both nuclear and mitochondrial loci may be useful for clarifying these differences.

The phylogenetic groupings observed with the mitochondrial sequences were similar to those obtained by Qin et al. (37) using IGS sequences, the difference being in the IGS analysis isolates $\mathrm{Cf} 45$ and $\mathrm{Ca} 63$ were nearly identical and grouped on the same clade (VdNC-II) with isolate GH101 yet in the mitochondrial analysis GH101 was clearly separated. Some of these same isolates were also examined by Bhat and Subbarao (4) using RAPD analysis and in general the grouping they observed was also seen in the haplotype analysis (the primary difference being a more distant relationship between MP89 and the clade containing SM113 and St91 in the mitochondrial analysis).

As noted above, there is good agreement in general between the phylogenetic groupings of VCGs derived from mitochondrial sequences and nuclear loci; however, there were several notable exceptions with several possible reasons contributing to this. The mitochondrial loci could be evolving at a different rate than the nuclear loci, particularly since the polymorphic mitochondrial sequences are in spacer regions and are not believed to be under selective constraints against mutations. It is also possible that rather than reproducing in a strictly clonal fashion that at some point in time there may have been recombination causing changes in VCG, leading to an uncoupling of the association between VCG and mitochondrial haplotype for some isolates. A more comprehensive phylogenetic analysis using both nuclear and mitochondrial loci for a wider collection of isolates fully characterized to VCG is needed to clarify if the mitochondrial phylogeny is congruent with the nuclear phylogeny. The genomic sequencing of $V$. dahliae (42) should facilitate the selection of additional nuclear loci that will provide further phylogenetic discrimination among isolates.

The results of this preliminary analysis suggest that mitochondrial haplotype classification can provide another marker for classification of field isolates that may provide additional separation of isolates grouped together using nuclear loci. However, a much larger sampling of isolates from a broader geographic area and diversity of hosts is needed before the utility of this analysis can be fully assessed. With the isolates examined in this study, the results of all five mitochondrial loci were used to assign haplotype classification, but depending on the number of haplotypes present in a particular location and the polymorphisms differentiating them it may be possible to use fewer loci. Based on the results presented in Table 1, data from regions 3 and 5 will separate all 15 haplotypes except H-V and H-IX and H-XII and $\mathrm{H}-\mathrm{XIII}$, but additional sequencing of region 1 will differentiate these haplotypes. With the genomic sequencing of $V$. dahliae isolate Ls17 in progress (42) there will be another mitochondrial genomic sequence available to compare to DQ531941 to identify additional regions that may be useful for differentiating haplotypes as well as enabling a more comprehensive phylogenetic analysis. One drawback for conducting haplotype analysis for a large number of samples is the amount of sequencing that would be required, but this can be reduced for the three larger amplicons (regions 3, 4, and 5) by redesigning the sequencing primers to focus on collecting data from the polymorphic regions only. Also, another approach that is less time consuming is melt curve analysis; this technique has been successfully used to determine haplotypes for Phytophthora ramorum and is more time efficient than sequencing (F. Martin and L. Radmer, unpublished data).

\section{ACKNOWLEDGMENTS}

The assistance of L. Radmer in conducting portions of the sequence analysis is gratefully acknowledged. Mention of trade names or commercial products in this manuscript is solely for the purpose of providing specific information and does not imply recommendation or endorsement by the U.S. Department of Agriculture.

\section{LITERATURE CITED}

1. Atallah, Z. K., Maruthachalam, K., du Toit, L., Koike, S. T., Davis, R. M., Klosterman, S. J., Hayes, R. J., and Subbarao, K. V. 2010. Population analysis of the vascular plant pathogen Verticillium dahliae detect recombination and transcontinental gene flow. Fungal Genet. Biol. 47:416-422.

2. Bell, A. A. 1994. Mechanisms of disease resistance in Gossypium species and variation in Verticillium dahliae. Pages 225-235 in: Proc. World Cotton Res. Conf. G. A. Constable and N. W. Forrester, eds. CSIRO, Melbourne, Australia.

3. Bhat, R. G., Smith, R. F., Koike, S. T., Wu, B. M., and Subbarao, K. V. 2003. Characterization of Verticillium dahliae isolates and wilt epidemics of pepper. Plant Dis. 87:789-797.

4. Bhat, R. G., and Subbarao, K. V. 1999. Host range specificity in Verticillium dahliae. Phytopathology 89:1218-1225.

5. Carder, J. H., and Barbara, D. J. 1991. Molecular variation and restriction fragment length polymorphisms (RFLPs) within and between six species of Verticillium. Mycol. Res. 95:935-942.

6. Chen, W. 1994. Vegetative compatibility groups of Verticillium dahliae from ornamental woody plants. Phytopathology 84:214-219.

7. Cherrab, M., Serrhini, M. N., and Charest, P. M. 2000. Characterization of Moroccan isolates of Verticillium dahliae Kleb using RAPD markers. J. Phytopathol. 148:243-249.

8. Clement, M., Posada, D., and Crandall, K. A. 2000. TCS: A computer program to estimate gene genealogies. Mol. Ecol. 9:1657-1660.

9. Collado-Romero, M., Mercado-Blanco, J., Olivares-García, C., and Jiménez-Díaz, R. M. 2008. Pathogenic analysis of Verticillium dahliae vegetative compatibility groups. Phytopathology 98:1019-1028.

10. Collado-Romero, M., Mercado-Blanco, J., Olivares-García, C., ValverdeCorredor, A., and Jiménez-Díaz, R. M. 2006. Molecular variability within and among Verticillium dahliae vegetative compatibility groups determined by fluorescent amplified fragment length polymorphism and polymerase chain reaction markers. Phytopathology 96:485-495.

11. Collins, A., Mercado-Blanco, J., Jiménez-Díaz, R. M., Olvares, C., Clewew, E., and Barbara, D. J. 2005. Correlation of molecular markers and biological properties in Verticillium dahliae and the possible origins of some isolates. Plant Pathol. 54:549-557.

12. Dobinson, K. F., Harrington, M. A., Omer, M., and Rowe, R. C. 2000. Molecular characterization of vegetative compatibility group $4 \mathrm{~A}$ and $4 \mathrm{~B}$ isolates of Verticillium dahliae associated with potato early dying. Plant Dis. 84:1241-1245.

13. Dobinson, K. F., Patterson, N. A., White, G. J., and Grant, S. 1998. DNA fingerprinting and vegetative compatibility analysis indicate multiple origins for Verticillium dahliae race 2 tomato isolates from Canada. Mycol. Res. 102:1089-1095.

14. Douhan, L. I., and Johnson, D. A. 2001. Vegetative compatibility and pathogenicity of Verticillium dahliae from spearmint and peppermint. Plant Dis. 85:297-302.

15. Eastburn, D. M., and Chang, R. J. 1994. Verticillium dahliae: A causal agent of root discoloration of horseradish in Illinois. Plant Dis. 78:496-498. 
16. Joaquim, T. R., and Rowe, R. C. 1990. Reassessment of vegetative compatibility relationships among strains of Verticillium dahliae using nitrate-nonutilizing mutants. Phytopathology 80:1160-1166.

17. Joaquim, T. R., and Rowe, R. C. 1991. Vegetative compatibility and virulence of strains of Verticillium dahliae from soil and potato plants. Phytopathology 81:552-558.

18. Katan, T. 2000. Vegetative compatibility in populations of VerticilliumAn overview. Pages 69-86 in: Advances in Verticillium Research and Disease Management. E. C. Tjamos, R. C. Rowe, J. B. Heale, and R. D. Fravel, eds. American Phytopathological Society, St. Paul, MN.

19. Klosterman, S. J., Atallah, Z. K., Vallad, G. E., and Subbarao, K. V. 2009. Diversity, pathogenicity and management of Verticillium species. Ann. Rev. Phytopathology 47:39-62.

20. Koike, M., Fujita, M., Nagao, H., and Ohshima, S. 1996. Random amplified polymorphic DNA analysis of Japanese isolates of Verticillium dahliae and V. albo-atrum. Plant Dis. 80:1224-1227.

21. Korolev, N., Pérez-Artés, E., Bejarano-Alcázar, J., Rodriguez-Juardo, D., Katan, J., Katan, T., and Jiménez-Díaz, R. M. 2001. Comparative study of genetic diversity and pathogenicity among populations of Verticillium dahliae from cotton in Spain and Israel. Eur. J. Plant Pathol. 107:443-456.

22. Lachqer, K., and Sedra, M. H. 2002. Characterization of Verticillium dahliae Kleb. isolates from Olea europaea using RAPD markers. Phytopathol. Mediterr. 41:170-178.

23. Librado, P., and Rozas, J. 2009. DnaSP v5: A software for comprehensive analysis of DNA polymorphism data. Bioinformatics 25:1451-1452.

24. Martin, F. N. 2008. Mitochondrial haplotype determination in the oomycete plant pathogen Phytophthora ramorum. Curr. Genet. 54:2334.

25. Mercado-Blanco, J., Rodríguez-Juardo, D., Parrilla-Araujo, S., and Jiménez-Díaz, R. M. 2003. Simultaneous detection of the defoliating and nondefoliating Verticillium dahliae pathotypes in infected olive plants by duplex nested PCR chain reaction. Plant Dis. 87:1487-1494.

26. Messner, R., Schweigkofler, W., Schweigkofler, M., Berg, G., and Prillinger, H. 1996. Molecular characterization of the plant pathogen Verticillium dahliae Kleb. using RAPD-PCR and sequencing of the $18 \mathrm{~S}$ rRNA gene. J. Phytopathol. 144:347-354.

27. Milne, I., Wright, F., Rowe, G., Marshal, D. F., Husmeier, D., and McGuire, G. 2004. TOPALi: Software for automatic identification of recombinant sequences within DNA multiple alignments. Bioinformatics 20:1806-1807.

28. Morton, A., Carder, J. H., and Barbara, D. J. 1995. Sequences of the internal transcribed spacer of the ribosomal RNA genes and relationships between isolates of Verticillium albo-atrum and $V$. dahliae. Plant Pathol. 44:183-190.

29. Morton, A., Tabrett, A. M., Carder, J. H., and Barbara, D. J. 1995. Subrepeat sequences in the ribosomal RNA intergenic regions of Verticillium albo-atrum and V. dahliae. Mycol. Res. 99:257-266.

30. Okoli, C. A. N., Carder, J. H., and Barbara, D. J. 1993. Molecular variation and sub-specific groupings within Verticillium dahliae. Mycol. Res. 97:233-239.

31. Okoli, C. A. N., Carder, J. H., and Barbara, D. J. 1994. Restriction fragment length polymorphism (RFLP) and the relationships of some host adapted isolates of Verticillium dahliae. Plant Pathol. 43:33-40.

32. Otero, L., Duchasse, D., and Miller, R. N. G. 2004. Variability in ribosomal DNA genic and spacer regions in Verticillium dahliae isolates from different hosts. Fitopatol. Bras. 29:441-446.

33. Pantou, M. P., Kouvelis, V. N., and Typas, M. A. 2006. The complete mitochondrial genome of the vascular wilt fungus Verticillium dahliae: A novel gene order for Verticillium and a diagnostic tool for species identification. Curr. Genet. 50:125-136.

34. Pérez-Artés, E., García-Pedrajas, M. D., Bejarano-Alcázar, J., and Jiménez-Díaz, R. M. 2000. Differentiation of cotton-defoliating and nondefoliating pathotypes of Verticillium dahliae by RAPD and specific PCR analysis. Eur. J. Plant Pathol. 106:507-517.

35. Posada, D. 2008. jModelTest: Phylogenetic model averaging. Mol. Biol. Evol. 25:1253-1256.

36. Pramateftaki, P. V., Antoniou, P. P., and Typas, M. A. 2000. The complete DNA sequence of the nuclear ribosomal RNA gene complex of Verticillium dahliae: Intraspecific heterogeneity within the intergenic spacer region. Fungal Genet. Biol. 29:19-27.

37. Qin, Q. M., Vallad, G. E., Wu, B. M., and Subbarao, K. V. 2006. Phylogenetic analysis of pathogenic isolates of Verticillium spp. Phytopathology 96:582-592.

38. Ramsay, J. R., Multani, D. S., and Lyon, B. R. 1996. RAPD-PCR identification of Verticillium dahliae isolates with different pathogenicity on cotton. Aust. J. Agric. Res. 47:628-693.

39. Strausbaugh, C. A. 1993. Assessment of vegetative compatibility and virulence of Verticillium dahliae isolates from Idaho potatoes and tester strains. Phytopathology 83:1253-1258.

40. Strausbaugh, C. A., Schroth, M. N., Weinhold, A. R., and Hancock, J. G. 1992. Assessment of vegetative compatibility and virulence of Verticillium dahliae tester strains and isolates from California potatoes. Phytopathology 82:61-68.

41. Subbarao, K. V., Chassot, A., Gordon, T. R., Hubbard, J. C., Bonello, P., Mullin, R., Okamoto, D., Davis, R. M., and Koike, S. T. 1995. Genetic relationships and cross pathogenicities of Verticillium dahliae isolates from cauliflower and other crops. Phytopathology 85:1105-1112.

42. The Broad Institute of the Massachusetts Institute of Technology and Harvard University. 2010. Verticillium Group Database. http://www. broad.mit.edu/annotation/genome/verticillium_dahliae/MultiHome.html

43. Usami, T., Ishigaki, S., Takashina, H., Matsubara, Y., and Amemiya, Y. 2006. Cloning of DNA fragments specific to the pathotype and race of Verticillium dahliae. J. Gen. Plant Pathol. 73:89-95.

44. Zeise, K., and von Tiedemann, A. 2002. Application of RAPD-PCR for virulence type analysis within Verticillium dahliae and V. longisporum. J. Phytopathol. 150:557-563. 